\title{
Status of India's immunization programme: Achieving goals of global vaccine action plan
}

\author{
Priti Chaudhary $^{1}$, Sourabh Saxena ${ }^{2, *}$, Sheenu Chaudhary ${ }^{3}$ \\ ${ }^{1}$ Consultant, UNICEF India, ${ }^{2}$ State Training Officer-RISE, ${ }^{3}$ Technical Officer, John Snow India, New Delhi, India
}

*Corresponding Author:

Email: drsaxenaaa@gmail.com

\begin{abstract}
In May 2012, World Health Assembly approved the Global Vaccine Action Plan (GVAP). The aim of GVAP is to achieve the Decade of Vaccines vision by delivering universal access to immunization. The mission of GVAP is to improve health by 2020 by extending full benefits of immunization to all people all over the world. Various indicators assessing the progress of the countries' immunization program are available on the GVAP portal along with annual data of these countries. The immunization programs of the countries can be evaluated and compared based on these indicators. Immunization programme of India is one of the largest programme in the world and caters to a a birth cohort of 2.6 million. In last decade, new vaccines like Pentavalent, Inactivated Polio Vaccine (IPV), Pneumococcal Conjugated Vaccine (PCV), Measles \& Rubella vaccine and rotavirus vaccine have been introduced along with new technology like electronic vaccine intelligence network (eVIN) to monitor the vaccine logistics. National Technical Advisory Group on Immunization (NTAGI) and Adverse Effect following Immunization (AEFI) surveillance establishment in India has further strengthened the program. The Universal Immunization Programme of India is moving in a direction that is consistent with the perspective of the global vaccine action plan (GVAP) making it possible for the country to achieve most of the targets laid down by GVAP by 2020.
\end{abstract}

Keywords: Global Vaccine Action Plan, Immunization, UIP, NTAGI, AEFI.

\section{Introduction}

Immunization programs are considered one of the most cost-effective health intervention. Over the past ten years, immunization has helped in saving millions of lives worldwide and helped in eradicating small-pox from the world, eliminating polio from most of the countries and measles and rubella from few of the countries. In recent years new vaccines like rotavirus vaccine, pneumococcal vaccine, HPV vaccine have been introduced but these vaccines are yet to be introduced in most of the countries in the world. Gavi, an international organization - a Global Vaccine Alliance is assisting countries in introducing new vaccines and strengthening of immunization programme. Gavi has supported India in introduction of various vaccines like Hep B, Pentavalent, Inactivated Polio Vaccine (IPV), Measles \& Rubella (MR) and Pneumococcal Conjugated Vaccine $(\mathrm{PCV})$ and expansion of rotavirus vaccine. ${ }^{1}$

With introduction of new vaccines and increasing momentum gained by Immunization Programmes, it became important to develop a framework which would help in strengthening and monitoring of immunization programmes in different countries of the world. In May 2012, the World Health Assembly approved, the Global Vaccine Action Plan (GVAP) to achieve the Decade of Vaccines vision by delivering universal access to immunization. The mission of GVAP is to improve health by by 2020 by extending the full benefits of immunization to all people around the world. ${ }^{2}$

Various indicators assessing the progress of the countries' immunization program are available on the GVAP portal along with annual data of these countries. The indicators measure progress against the GVAP goals to: (1) improve routine immunization to meet vaccination coverage and equity targets; (2) eradicate polio, eliminate measles, rubella and maternal and neonatal tetanus; (3) introduce new and improved vaccines; and (4) spur research and development for the next generation of vaccines and technologies. ${ }^{3}$

The Universal Immunization Programme (UIP) has been operational in India since 1985 and is expected to provide the vaccines to 26 million children annually. The objectives of UIP are to increase full immunization coverage to more than $90 \%$, establish a strengthened cold chain and vaccine logistics system, improve the quality of services and introduce a district-wise system for monitoring of immunization programme performance, and achieve self-sufficiency in vaccine production and management. The main goal of the UIP is to reduce mortality and morbidity due to vaccine preventable diseases (VPD). The programme which initially offered protection against six vaccine preventable diseases, today offers protection from twelve vaccine preventable diseases and is working towards the goal of protecting each and every child in the country from vaccine preventable diseases. ${ }^{4}$

GVAP has well defined indicators to measure the progress of immunization programme in the countries. In this paper, we are making an attempt to evaluate the current status of India's immunization programme as per the goals and objectives indicators defined by GVAP.

\section{Global Vaccine Action Plan Monitoring Indicators}

There are five goals and each goal have its own indicators to measure the progress of immunization programme. If these goals are achieved by all the 
countries of the world then hundreds of millions of vaccines preventable diseases (VPD) cases and millions of future deaths will be averted by the end of 2002 and economic productivity will be gained. Also, immunization will contribute to the Millennium Development Goal 4 target for reducing child mortality and Sustainable Development Goal 3 target i.e. By 2030, end preventable deaths of newborns and children under 5 years of age, with all countries aiming to reduce neonatal mortality to at least as low as 12 per 1000 live births and under-5 mortality to at least as low as 25 per 1000 live births. ${ }^{5}$

Table 1: Goals of the decade of Vaccines ${ }^{3}$

\begin{tabular}{|l|l|l|l|}
\hline \multicolumn{1}{|c|}{ S. No } & \multicolumn{1}{|c|}{ Goal } & \multicolumn{1}{c|}{ Indicators } \\
\hline 1 & Reduced Mortality & a) Reduce under-five mortality rate \\
& b) Integration of health care interventions, immunization \\
& Polio Eradication & a) Interrupt wild poliovirus transmission globally \\
\hline 2 & Elimination Targets & b) Certification of poliomyelitis eradication
\end{tabular}

\section{Status of India's Immunization Programme as per GVAP Indicators}

Reduced Mortality: During the past decade, Universal Immunization Programme has made tremendous progress in preventing morbidity and mortality due to vaccine preventable diseases. In addition, to the BCG, DPT, OPV and Measles vaccines, Hepatitis B vaccine was introduced in country in 2002-03 and expanded to the entire country by 2010 to protect children from liver diseases. Additionally, in 2010, in order to eliminate deaths due to measles, Measles vaccine's second dose was introduced in Universal Immunization Programme. As per WHO data, with the introductions of measles second dose, measles cases and deaths decreased by nearly $50 \%$ from 2011 to $2013 .{ }^{4}$ There has been huge spurge in reduction of under-five mortality rate (U5MR) in country i.e. U5MR declined from 126 per 1,000 live births in 1990 to 39 per 1,000 live births in $2016 .{ }^{6}$ How much of this can be contributed to immunization programme, cannot be said as India still lacks a robust VPD surveillance system.

Polio Eradication and Elimination Targets: On 27th March, 2014, India achieved one of the major milestone in immunization program and certified as Polio free. .? In May 2015, the country achieved one more milestone by getting validated for Maternal and Neonatal Tetanus elimination which was well before the global target date of December 2015. This was a monumentous achievement in the country's efforts to achieve universal health care and address health inequities. ${ }^{8}$ With introduction of Measles \& Rubella (MR) Vaccine, India has shown it's commitment towards the goal of measles elimination and rubella/ congenital rubella syndrome (CRS) control. MR vaccine was launched on $5^{\text {th }}$ February 2017 and is being introduced in the country via campaign following which it will be given in routine immunization in place of two doses of measles vaccine. As of October 2017, 33.5 million children have already received the MR vaccine through this campaign. ${ }^{9}$ However, with delay in introduction of MR vaccine it is difficult to say that India will be able to achieve the goal by 2020 .

New Vaccine Introduction: In addition to MR vaccine, India has also introduced rotavirus vaccine and pneumococcal conjugated vaccine in select states with 
plan to expand the vaccines in entire country in phased manner. ${ }^{4}$ With launch of new vaccines, it becomes even more important to have a robust vaccine stock and quality monitoring system. An Electronic Vaccine Intelligence Network (eVIN) was launched by Ministry of Health and Family Welfare, Government of India. The main aim of eVIN is to strengthen the vaccine supply chain to ensure equity in vaccine availability. It is an indigenously technology system developed in India and digitizes vaccine stocks and is used to monitor the temperature of the cold chain through a smartphone application. eVIN is a valuable technology and support UIP by providing real-time information on vaccine stocks and movement, and storage temperatures across all cold chain points in the country. Till now, eVIN is fully functional in twelve states of the country and is being expanded to the remaining states. ${ }^{10}$

Immunization Coverage: The full immunization coverage (FIC) in the country has progressed from $35.5 \%$ in $1992-93$ (NFHS-1) to $62 \%$ in $2015-16$ (NFHS4). However, India is yet to achieve the aim of $90 \%$ national coverage and $80 \%$ in every district. With implementation of Mission Indradhanush in the country, it is expected that immunization coverage will definitely increase but it is highly unexpected that aim of $90 \%$ coverage would be achieved by $2020 .{ }^{11}$

Fig. 2: GVAP strategic objectives indicators

\begin{tabular}{|c|c|c|c|}
\hline S.No & Strategic Objective & & Indicators \\
\hline 1 & Country's ownership & a) & $\begin{array}{l}\text { Presence of an independent technical advisory group that } \\
\text { meets the defined criteria }\end{array}$ \\
\hline 2 & Research \& development & $\begin{array}{l}\text { a) } \\
\text { b) } \\
\text { c) } \\
\text { d) } \\
\text { e) } \\
\text { f) } \\
\text { g) }\end{array}$ & $\begin{array}{l}\text { Progress towards a universal influenza vaccine } \\
\text { Progress towards development of HIV vaccines } \\
\text { Progress towards institutional and technical capacity to } \\
\text { carry out vaccine clinical trials } \\
\text { Progress towards development of TB vaccines } \\
\text { Progress towards development of malaria vaccines } \\
\text { Licensure and launch of at least one platform delivery } \\
\text { technology } \\
\text { Licensure and launch of vaccine or vaccines against one } \\
\text { or more major currently non-vaccine preventable } \\
\text { diseases }\end{array}$ \\
\hline 3 & Vaccine hesitancy & a) & $\begin{array}{l}\text { Percentage of countries that have assessed the level of } \\
\text { hesitancy towards vaccination at the national or } \\
\text { subnational level in the previous five years } \\
\text { Reasons for vaccine hesitancy }\end{array}$ \\
\hline 4 & Vaccine safety & h) & $\begin{array}{l}\text { Number of AEFI reported by country per } 100000 \\
\text { surviving infants. The target is currently set at a ratio of } \\
10 \text { based on an empirical analysis of JRF data since } 2000\end{array}$ \\
\hline 5 & Vaccine surveillance & a) & $\begin{array}{l}\text { Number of Member States with case-based surveillance } \\
\text { for vaccine-preventable diseases: invasive bacterial } \\
\text { vaccine-preventable diseases and rotavirus }\end{array}$ \\
\hline 6 & Vaccine supply & $\begin{array}{l}\text { a) } \\
\text { b) }\end{array}$ & $\begin{array}{l}\text { Number of vaccines that have either been re-licensed or } \\
\text { licensed for use in a controlled-temperature chain at } \\
\text { temperatures above the traditional } 2-8 \text { degree Celsius } \\
\text { Availability of vaccines for routine immunization at } \\
\text { national level }\end{array}$ \\
\hline 7 & Financing & a) & $\begin{array}{l}\text { Number of vaccine delivery technologies (devices and } \\
\text { equipment) that have received who prequalification } \\
\text { All Member States commit to immunization as a priority: } \\
\text { domestic expenditures for immunization per person } \\
\text { targeted } \\
\text { Vaccine Price Indicator: Annual average or unit vaccine } \\
\text { prices } \\
\text { Vaccine Price Indicator: Number of countries sharing } \\
\text { price information through the V3P platform by WHO } \\
\text { region }\end{array}$ \\
\hline
\end{tabular}


Status of India's Immunization Programme as per GVAP Strategic Objectives Indicators

As per the WHO 2018 data on Immunization System indicators ${ }^{12}$, India is successfully working towards achieving all the indicators. India has an independent technical advisory group that meets the criteria defined by WHO, regularly reports to WHOUNICEF the reasons for vaccine hesitancy, has established Vaccine Preventable Disease (VPD) surveillance system which is being strengthened to achieve case-based surveillance for all the VPDs, vaccine supply is being monitored using eVIN which is being expanded to all the States/UTs of the country, Adverse Event Following Immunization (AEFI) surveillance system has been established and Govt uses domestic funds to buy most of the vaccines and operational part of the immunization program. However, India is yet to share the price information through the V3P platform and needs to strengthen the VPD and AEFI surveillance systems to achieve the targets set by GVAP. Country's Ownership and Research \& Development: In 2001, the National Technical Advisory Group on Immunization (NTAGI), ${ }^{13}$ was established by the Ministry of Health \& Family Welfare, Govt. of India. NTAGI's main role is to provide advice and guide the national government regarding the technical issues of vaccine introduction in UIP. The NTAGI group meets at least twice a year and evaluate and guide government on various policy and technical decisions of immunization program in the country. The Secretary (Health \& Family Welfare) has been designated as the Chair and the Deputy Commissioner (Immunization Division) as its Member-Secretary and NTAGI has members from a various fields relevant to the program. Over the years, the role of the NTAGI (and consequently the membership) has evolved to meet the changing requirements. Other issues pertaining to immunization have also been taken up for ongoing discussions such as improving coverage and access, promoting vaccine security, monitoring of adverse events following vaccinations, vaccines beyond childhood (like human papilloma virus, seasonal influenza and meningitis vaccines), public-private partnerships in the UIP, and measuring and monitoring the impact of immunizations. The NTAGI in India meets all the six process indicators agreed upon by WHO and its partners. These are (1) formal written terms of reference; (2) legislative or administrative basis for the advisory group; (3) at least one meeting per year; (4) at least one five different areas of expertise represented among core members; (5) mandatory disclosure of any conflict of interest; (6) circulation of the agenda and background documents at least one week prior to meetings. Since the establishment of NTAGI in 2001, seven new vaccines have been introduced in UIP. At present India is conducting third highest number of vaccine clinical trials and has been ranking consistently higher than any European country over the four years of reporting. During the period of
2012-2016, India conducted a total of 144 vaccine clinical trials which are conducted both by public and private organizations. ${ }^{14}$

Vaccine Hesitancy: Though new vaccines are being introduced in the country, India is yet to achieve the FIC of $90 \%$. To achieve the aim of $90 \%$ FIC, it is important to understand the reasons for low immunization coverage. As per Coverage Evaluation Survey (CES), 2009 major reasons for low immunization coverage is information gap and about $8 \%$ of parents don't get their children vaccinated due to fear of side effects. Even the 2016 WHO-UNICEF JRF ${ }^{12}$ data reveals that worldwide, the most frequently listed determinants for vaccine hesitancy continue to be: a) lack of knowledge and awareness of vaccination and its importance; b) riskbenefit issues of the vaccine, in particular concerns around vaccine safety; c) religion, culture, gender and socioeconomic issues, in particular religious reasons.

Vaccine Safety: In view of these points it becomes important to have robust AEFI surveillance in the country. The AEFI surveillance was started in India in 1988 but National AEFI guidelines were developed in 2005 and National AEFI committee was formed in $2008 .{ }^{15}$ During 2012-2014, approximately 1,346 million doses of all antigens were given in the country while only 1,759 cases of serious AEFIs were reported indicating that the AEFI events are well within the expected rates though there is an overall need to further improve surveillance. ${ }^{16}$ As per 2015 WHO data India is reporting $<10$ AEFI cases per 100,000 surviving infants whereas America and European regions are reporting $\geq 10$ AEFI per 100000 surviving infants. ${ }^{17}$ This clearly indicates that we need to strengthen the AEFI surveillance system in India.

Vaccine Surveillance: In addition to the AEFI surveillance system it is also important to have a good VPD surveillance system as it gives information about the disease burden which is required before introduction of any vaccine. Also, it gives information about the impact of vaccine introduction in decreasing the case burden of VPDs. In India, Central Bureau of Health Intelligence (CBHI) is the main source of information for vaccine preventable disease cases and deaths. The data is provided state wise, however no information is provided on other epidemiological indicators like age, gender, ethnicity, vaccination status etc. The data reported by $\mathrm{CBHI}$ is essentially rural cases that come to the attention of PHCs, which would be only a small fraction of the total. Even such statistics are not validated and have poor reliability. The other source of data is from Integrated Disease Surveillance Program (IDSP) which is an outbreak-based surveillance, hence not giving any information on the cases. With the establishment of IDSP it has helped in detecting various VPDs outbreaks in the country. Even now case-based surveillance is not available in India except the AFP surveillance and Measles-Rubella surveillance which is conducted by WHO. Recently WHO has established 
laboratory supported VPD surveillance in country which would collect data on diphtheria, pertussis and neonatal tetanus. It has currently been established in Haryana, Bihar and Kerala and will be expanded to entire country in coming future. In addition to this India has also established Indian Rotavirus Strain Surveillance Network. It is a comprehensive multicenter, multi-state hospital-based surveillance network with centers in eight cities of the country. However, the information about rotavirus cases and other VPDS cases is not shared with WHO IB-VPD Surveillance Network and WHO Rotavirus Surveillance Network. ${ }^{18}$

Vaccine Supply and Financing: Vaccine safety and security is the most important part of the immunization programme. It is important that there should not be any stock outs and only safe vaccine should be given to beneficiaries. The Central Drugs and Standards Control Organization (CDSCO) is the National Regulatory Authority (NRA) in India. The main role of CDSCO is to givegives approval for the vaccines that are introduced in the country. It also registers and controls the quality of imported vaccines, is the main authority to grant permission to conduct clinical trials. . The manufacturers of vaccines are provided license by CDCSO which also act as the Central License Approving Authority (CLAA) for the manufacturer of vaccines and coordinates the activities of the States. . India is a vaccine manufacturing country and most of the vaccines used in UIP are manufactured locally. Public Sector Units (PSUs) are mostly encouraged to manufacture vaccines locally and thus ensuring availability of vaccines at low cost. In India, the vaccine purchasing and delivery system in is well developed. Govt. of India calculates the long-term projections of new and unused vaccines well in advance and share the same with the vaccine manufacturing industry in order to shape the industry and to maintain vaccine security. This in turn also helps in avoidance of the stock-out situations or wastage of vaccines. ${ }^{12}$ The country has not experienced any vaccine stock-outs in $2016 .{ }^{20}$ In the country, both WHO pre-qualified and nonqualified vaccine manufacturers are present. However, vaccine is supplied by all the manufacturers as India's birth cohort is quite large. This helps in constant supply of vaccines at a low cost and also promotes competition among the various manufacturers. During the procurement of vaccines, Govt. of India gives the tender to the lower bidder. At present, Govt. of India procures vaccines for Universal Immunization Programme and private hospitals/practitioners procure vaccines directly from the vaccine manufacturers. However, procurement price of vaccine for either sector is in-evident to each other. Therefore, a regulatory database giving information on vaccine procurement price is a need. ${ }^{19}$

\section{Conclusion}

India has made great progress in last decade, and even though some challenges still remain, the programme has moved in a direction that is consistent with the perspective of the global vaccine action plan (GVAP). With certification of polio free country and maternal and neonatal tetanus elimination, the program has made a global achievement. With introduction of MR vaccine, the country has shown its commitment towards measles and rubella elimination.

New vaccines and technology have been introduced in the country which will further help in strengthening of the programme. With implementation of Mission Indradhanush and recent introduction of Intensified Mission Indradhanush, it is expected that full immunization coverage in the country will increase. The country is constantly working towards achieving a wellestablished AEFI and case-based VPD surveillance system.

While the impact of these efforts is yet to be seen, there is little doubt that the Immunization programme in India has made impressive strides towards the achievement of its comprehensive objective still has a chance to achieve most of the targets laid down by GVAP by 2020 .

\section{References}

1. Gavi India Factsheet. Available from: https://www.gavi.org/country/india/ Accessed on 6 June 2018

2. Immunization, Vaccines and Biologicals. Global Vaccine Action Plan Available from: http://www.who.int/immunization/global_vaccine_action _plan/en/ Accessed on 6 June 2018

3. Global Vaccine Action Plan Indicator Portal. [Updated August2017) Available from: http://apps.who.int/gho/cabinet/gvap.jsp Accessed on 6 June 2018

4. Routine Immunization Background Note. Available from: https://mohfw.nic.in/showfile.php?lid=4248 Accessed on 6 June 2018

5. Sustainable Development Goals (SDGs) SDG 3: Ensure healthy lives and promote wellbeing for all at all ages. Available from: http://www.who.int/sdg/targets/en/ Accessed on 6 June 2018

6. Child Survival: Current Status + Progress. Available from: https://data.unicef.org/topic/child-survival/underfive-mortality/. Accessed on 28 March 2018

7. Immunization: India three years polio-free Polio eradication and certification. Available from: http://www.searo.who.int/immunization/topics/polio/erad ication/sea-polio-free/en/ Accessed on 6 June 2018

8. Sidhu J, Dewan P, Gupta P. Maternal and Neonatal Tetanus: A Journey into Oblivion. Indian Pediatr [Internet] 2015 [cited 2018 March 24] 53: 1057-1061. Available from: http://www.indianpediatrics.net/dec2016/dec-10571062.htm

9. Measles \& Rubella Vaccination Campaign. Available from: http://measlesrubella.in/ Accessed on 6 June 2018

10. Improving Efficiency of Vaccination Systems in Multiple States. Available from: http://www.in.undp.org/content/india/en/home/operations /projects/health/evin.html. Accessed on 27 March 2018

11. Mission Indradhanush Available from: http://www.missionindradhanush.in/ Accessed on 6 June 2018. 
12. WHO vaccine-preventable diseases: monitoring system. 2018 global summary. [Updated 2018 June 1] Available from:

http://apps.who.int/immunization_monitoring/globalsum mary/indicators?ir\%5Bc\%5D $\% 5 \mathrm{~B} \% 5 \mathrm{D}=\mathrm{IND} \& \mathrm{ir} \% 5 \mathrm{Ba} \%$ $5 \mathrm{D}=$ on $\&$ commit $=\mathrm{Ok}+$ with+the+selection Accessed on 6 June 2018

13. John TJ. India's National Technical Advisory Group on Immunisation. Vaccine. 2010 Apr 19;28 Suppl 1:A88-90. doi: 10.1016/j.vaccine.2010.02.041.

14. Global Vaccine Action Plan Secretariat Annual Report 2016 Chapter 10: Research and Development p196. Available from: http://www.who.int/immunization/global_vaccine_action _plan/gvap_secretariat_report_2016.pdf Accessed on 6 June 2018

15. AEFI surveillance and operational guidelines. MoHFW, Govt. of India, 2010

http://www.pbhealth.gov.in/Immunization/AEFI_Guideli nes.pdf

16. Causality assessment report of 367 reported Serious Adverse Events Following Immunization (AEFI) cases, approved by National AEFI Committee. Available from: https://mohfw.gov.in/sites/default/files/256999353614297 85864_0.pdf Accessed on 6 June 2018.

17. Global Vaccine Action Plan Secretariat Annual Report 2016 Chapter 10: Research and Development p167. Available from: http://www.who.int/immunization/global_vaccine_action _plan/gvap_secretariat_report_2016.pdf Accessed on 6 June 2018

18. Immunization Handbook for Medical Officers, MoHFW, Govt. of India, 3rd ed. India; 2016. 223-5 p.

19. National Vaccine Policy, MoHFW, Govt. of India, 2011 April Available from:

https://mohfw.gov.in/sites/default/files/1084811197.pdf Accessed on: 6 June 2018. 